6. N. Smirnov, Sur les ecarts de la courbe de distribution empirique, Mat. Sb. (N.S.) 6 (48) (1939), 3-26.

7. Goro Ishii, On the exact probabilities of Renyi's tests, Ann. Inst. Statist. Math. Tokyo 11 (1959), 17-24.

8. Li-Chien Chang, On the ratio of an empirical distribution function to the theoretical distribution function, Acta Math. Sinica 5 (1955), 347-368; English transl., Selected Transl. Math. Statist. and Prob., Vol. 4, pp. 17-38, Inst. Math. Statist. and Amer. Math. Soc., Providence, R. I., 1963.

9. S. C. Tang, Some theorems on the ratio of empirical distribution to the theoretical distribution, Pacific J. Math. 12 (1962), 1107-1114.

Princeton University

\title{
BANACH ALGEBRAS OF SCALAR-TYPE ELEMENTS
}

\section{BERTRAM WALSH}

In [5] H. Schaefer asked whether a locally convex algebra having the property that each of its elements is "spectral" (i.e. can be represented as the integral of some measurable function with respect to some spectral measure [is of "scalar type" in the sense of Dunford]) must necessarily be a commutative algebra. This note answers the question only for Banach algebras, but shows that the answer is affirmative under a hypothesis less restrictive than that which Schaefer suggests, and also (via a theorem of Katznelson [3]) that in fact a Banach algebra satisfying this hypothesis is automatically isomorphic to $\mathrm{e}(M), M$ its maximal ideal space. The result may also be viewed then as a variant of Katznelson's, in which commutativity and semisimplicity are not required $a$ priori.

For simplicity's sake the presence of an identity is assumed in all algebras discussed below, and homomorphisms are assumed to carry identities to identities. The modifications necessary to dispense with these assumptions are straightforward.

Let $\mathfrak{A}$ be a real or a complex Banach algebra. We shall say that an element $a \in \mathfrak{A}$ is prescalar under the following circumstances:

(1) If $\mathfrak{A}$ is a real Banach algebra, we require that the spectrum of $a$ (as defined for real Banach algebras via complexification-see [4, p. 28]) be real, and that there be a homomorphism $h_{a}: \mathfrak{C}_{R}(\sigma(a)) \rightarrow \mathfrak{A}$ (where as usual $\mathfrak{C}_{R}(\sigma(a))$ is the sup-norm algebra of continuous real-

Received by the editors November 2, 1964. 
valued functions on $\sigma(a)$ ) which sends the identity function on $\sigma(a)$ to $a$; letting $z$ denote the identity function, we can write this $h_{a}(z)=a$.

(2) If $\mathscr{Q}$ is a complex Banach algebra, we require that there be a homomorphism $h_{a}: \mathfrak{e}(\sigma(a)) \rightarrow \mathfrak{A}$ for which $h_{a}(z)=a$, where $z$ is the identity function on $\sigma(a)$. (Of course $z$ here is not the ring identity of the continuous-function ring, but the function for which $z(\lambda)=\lambda$.)

For example, if $a$ is a scalar-type element of $\mathcal{L}(E)$ where $E$ is a Banach space, then $a$ is a prescalar element of $\mathscr{L}(E)$ in the uniform norm, as in [1].

It is immediate that a prescalar element is a topological nilpotent if and only if it is zero, because if $\sigma(a)=\{0\}$, then the identity function and the zero element of $\mathfrak{C}(\sigma(a))$ (or $\mathfrak{C}_{R}(\sigma(a))$ respectively) are the same, so $a=h_{a}(z)=h_{a}(0)=0$. As a consequence we have the

Proposition. If $\mathfrak{A}$ is a Banach algebra each of whose elements is prescalar, then for each $a \in \mathfrak{A}$ any homomorphism $h_{a}$ as in the definition of prescalarity is automatically continuous.

Indeed, since the only topological nilpotent in $\mathscr{A}$ is zero, the closure of $h_{a}[\mathfrak{e}(\sigma(a))]$ (or its real counterpart) in $\mathfrak{A}$ is semisimple [4, p. 57]. Consequently $h_{a}$ is continuous by $[4, \mathrm{p} .75] .^{1}$

One may thus safely assume $h_{a}$ continuous in the following

Proposition. If $a$ is a prescalar element of $\mathfrak{A}$ for which a homomorphism $h_{a}$ rendering a prescalar is continuous, then whenever $\Im$ is a closed one-sided ideal of $\mathfrak{A}$ with $a^{k} \in \mathcal{Y}$ for some $k, a \in \Im$ also.

Indeed, consider the closed ideal $h_{a}^{-1}[\Im] \subseteq \mathfrak{e}(\sigma(a)$ ) (or its real counterpart). The closed ideals in $\mathfrak{C}(\sigma(a))$ are precisely the sets of functions vanishing on fixed closed subsets of $\sigma(a)$ (i.e. are the kernels of their hulls; cf. [4, p. 193]). By hypothesis $z^{k} \in h_{a}^{-1}[\Im]$, and $z^{k}$ and $z$ have the same set of zeros, whence $z \in h_{a}^{-1}[\Im]$, or $a=h_{a}(z) \in \Im$.

General information on elementary Jacobson ring structure theory, particularly the density theorem, and the relations of the algebraic structure to the topological in Banach algebras (on which the proof of the next theorem depends) may be found in [2] and [4].

THEOREM. Let $\mathfrak{A}$ be a Banach algebra each of whose elements is prescalar. Then $\mathfrak{A}$ is semisimple and commutative.

Proof. We have already seen that $\mathfrak{A}$ can have no topologically nilpotent elements; since every element of the radical of an algebra has

1 The proof that these homomorphisms have closed graphs is quite straightforward; the reader may find a direct proof in less time than it takes to reach for his copy of [4]. 
zero spectrum, $\mathfrak{A}$ must be semisimple. Now let $\mathfrak{R}$ be a maximal left ideal of $\mathfrak{A}$ and $\mathfrak{A}-\mathfrak{R}$ the difference Banach space; if $\Delta$ is the ring of those endomorphisms of the additive group of $\mathfrak{A}-\mathfrak{R}$ (no topology) which commute with all the operators induced on $\mathfrak{A}-\mathfrak{l}$ by the left regular representation of $\mathfrak{A}$ (i.e. the ring of $\mathfrak{A}$-module endomorphisms of $\mathfrak{A}-\mathfrak{l})$, then Schur's lemma says that $\Delta$ is a division ring and the Jacobson density theorem says that if $v_{1}, v_{2}$ are $\Delta$-linearly independent elements of $\mathfrak{A}-\mathfrak{l}$ and $w_{1}, w_{2}$ are any elements of $\mathfrak{A}-\mathfrak{l}$, then there exists $a \in \mathfrak{A}$ for which $a_{\mathbb{R} v_{i}}=w_{i}, i=1,2$, where $a_{\mathbb{q}}$ is the left regular representation of $\mathfrak{A}$ on $\mathfrak{A}-\mathfrak{R} .^{2}$ It follows that if all elements of $\mathfrak{A}$ are prescalar, then $\mathfrak{A}-\mathfrak{R}$ can have $\Delta$-dimension at most 1 , thus exactly 1 . For if $v_{1}$ and $v_{2}$ were $\Delta$-linearly independent in $\mathfrak{A}-\mathfrak{l}$, there would exist $a \in \mathfrak{A}$ with $a_{\mathbb{Q} v_{1}}=v_{2}, a_{\mathbb{Q} v_{2}}=0$. The left regular representation of $\mathfrak{A}$ on $\mathfrak{A}-\mathfrak{R}$ is continuous, so the annihilator $\mathfrak{Y}=\left\{b \mid b \in \mathfrak{A}, b_{\mathfrak{Q}} v_{1}=0\right\}$ of $v_{1}$ in $\mathfrak{A}$ is a closed left ideal, and since $\left(a^{2}\right)_{\mathbb{R}} v_{1}=a_{\mathbb{q}}\left(a_{\mathbb{q}} v_{1}\right)=a_{\mathbb{q}} v_{2}=0, a^{2} \in \Im$. By the proposition above, $a \in \Im$ also, so $v_{2}=a_{\varepsilon} v_{1}=0$ contrary to its choice.

Thus $\mathfrak{A}$ is represented on $\mathfrak{A}-\mathfrak{R}$ as an algebra of linear transformations of a one-dimensional vector space; since this algebra is transitive on the nonzero vectors of $\mathfrak{A}-\mathfrak{R}$, it contains an inverse for each of its nonzero elements, i.e. is a normed division algebra, hence is the reals, complexes or quaternions. If $\mathfrak{A}$ is a complex algebra the complex numbers are the only possibility for this representation; if $\mathfrak{A}$ is a real algebra and were represented as either the complexes or the quaternions, then there would exist an $a \in \mathfrak{A}$ with $\left(a_{\mathbb{R}}\right)^{2}=-1$, contrary to the invertibility of $1+z^{2}$ in $\mathfrak{C}_{R}(\sigma(a))$. Thus $\mathfrak{A}$ is represented on $\mathfrak{A}-\mathfrak{R}$ as the reals when $\mathfrak{A}$ is real and as the complexes when $\mathfrak{A}$ is complex; in either case it is represented as a commutative algebra, and there being sufficiently many of these representations to distinguish elements of $\mathfrak{A}, \mathfrak{A}$ is commutative.

COROLlaRy. If $\mathfrak{A}$ is a real Banach algebra each of whose elements is prescalar, then every maximal ideal of $\mathfrak{A}$ is real, i.e. its quotient ring is the real numbers.

Thus under the hypotheses of the theorem each maximal ideal of $\mathfrak{U}$ can be identified with a homomorphism of $\mathfrak{A}$ into its scalar field, and $\mathfrak{A}$ can be identified with its Fourier transform algebra $\mathfrak{U}^{\wedge}$ on the compact Hausdorff space $M$ of such homomorphisms. Prescalarity of all elements of $\mathfrak{A}$ implies that continuous functions operate on $\mathfrak{A}^{\wedge}$ in the sense of Katznelson [3], since for $a \in \mathfrak{A}$ and $m \in M$ one has

2 Actually $\Delta$ must already be one of the three normed real division algebras [4, p. 61], but this fact plays no part in what follows. 
$\left[h_{a}(f)\right]^{\wedge}(m)=m\left(h_{a}(f)\right)=\left[h_{a}^{*}(m)\right](f)$; but $h_{a}^{*}(m)$ is evaluation at $\left[h_{a}^{*}(m)\right](z)=m\left(h_{a}(z)\right)=m(a)=a^{\wedge}(m) \in \sigma(a)$, so $\left[h_{a}(f)^{\wedge}(m)\right.$ $=f\left(a^{\wedge}(m)\right)$ for any $m \in M$, and $h_{a}(f)$ is the result of $f$ operating on $a$ in the sense of [3]. Thus by the principal theorem of [3] (which is stated for complex algebras but proved for real ones, so applicable in both cases) we have

THEOREM. If $\mathscr{A}$ is a Banach algebra each of whose elements is prescalar, then $\mathfrak{A}$ is commutative and semisimple, and isomorphic to $\mathfrak{C}_{\mathbb{R}}(M)$ or $\mathrm{C}(M), M$ the space of maximal ideals of $\mathfrak{A}^{\mathrm{3}}$

\section{REFERENCES}

1. N. Dunford, Spectral operators, Pacific J. Math. 4 (1954), 321-354.

2. I. Herstein, Theory of rings, Univ. of Chicago Math. Lecture Notes, Spring 1961.

3. Y. Katznelson, Algebres caracterisees par les fonctions qui operent sur elles, C. R. Acad. Sci. Paris 247 (1958), 903-905.

4. C. Rickart, General theory of Banach algebras, Van Nostrand, New York, 1960.

5. H. Schaefer, Convex cones and spectral theory, Proc. of Symposium on Convexity, Amer. Math. Soc., Providence, R. I., 1963.

6. - Spectral measures in locally convex algebras, Acta Math. 107 (1962), 125-173.

\section{University of California, Los Angeles}

The referee has pointed out that this theorem and [1, Theorem 18] imply the existence of a simultaneous resolution of the identity for $\mathfrak{A}$ if $\mathfrak{U}$ is an algebra of bounded linear operators on a reflexive Banach space. The extension of this fact to weakly sequentially complete locally convex spaces follows from the principal results of [6]. 